\title{
Proof that every rational algebraic equation has a root
}

By Professor A. C. Dixon

(Read 10th January, 1908. Received, same date.)

The following arrangement of the proof of this theorem could, I think, be given at a comparatively early stage, even if the necessary case of De Moivre's theorem had to be proved as an introductory lemma.

Let $u, v$ be two rational integral algebraic functions of $x, y$ with real coefficients, and let $c$ be a simple closed contour in the plane. As the point $(x, y)$ travels round $c$ let those changes in the sign of $u$ that take place when $v$ is positive be marked and let $(u, v ; c)$ denote the excess in number among these of changes from + to over changes from - to $+^{*}$.

If $c$ is deformed continuously, $(u, v ; c)$ will not be changed except (1), when $c$ passes over a point where $u, v$ both vanish, (2), when there is a change in the number of points where $c$ meets one of the curves $u=0, v=0$. In case (1) there will generally be a change in the value of $(u, v ; c)$ since a change of sign in $u$ on $c$ will pass from the part of $c$ where $v$ is negative to that where $v$ is positive, or conversely.

In case (2) suppose $c$ to be deformed so that the number of its intersections with the curve $u=0$ is increased. The increase must be an even number since both curves are continuous and endless. The sign of $v$ is constant in the neighbourhood unless we are dealing with a case under ( 1 ) and since the new changes in sign of $u$ are alternately +- and -+ there is no effect on $(u, v ; c)$ : similarly if the number of intersections with $u=0$ is decreased.

If the number of intersections of $c$ with the curve $v=0$ is altered, it must again be by an even number and there will be no change in $(u, v ; c)$ unless $u=0$ at the same place as $v$ when the case falls under (1).

Hence the deformation of $c$ produces no effect on $(u, v ; c)$ unless c passes over a point where $u=0=v$. In particular, if $c$ does not contain such a point, it can be made to shrink up to a small contour in a neighbourhood where $u, v$ are of constant signs and $(u, v ; c)$ being unaffected by this process must be 0 throughout.

* It is not hard to see that $(v, u ; c)=(-u, v ; c)=-(u, v ; c)$ etc. 
Now take $u, v$ to be given by the equation $f(z)=u+\imath$ where $z=x+\iota y$ and $f(z)=z^{n}+\left(a_{1}+\iota b_{1}\right) z^{n-1}+\left(a_{2}+\iota b_{2}\right) z^{n-2}+\ldots:$ let $c$ be a circle with centre at the origin and radius $R$, so that on $c$ $z=\mathbf{R}(\cos \theta+i \sin \theta)$, and $\theta$ runs from 0 to $2 \pi$.

We have by De Moivre's theorem $u=\mathrm{R}^{n} \cos n \theta+\mathrm{R}^{n-3}\left(a_{1} \cos \overline{-1} \theta-b_{1} \sin \overline{n-1} \theta\right)+$ lower powers of $\mathrm{R}$, $v=\mathbf{R}^{n} \sin n \theta+\mathbf{R}^{n-1}\left(a_{1} \sin \overline{n-1} \theta+b_{1} \cos n-1 \theta\right)+$ lower powers of $\mathbf{R}$. The sum of all the terms after the first, either in $u$ or $v$, is not greater than the sum of $a_{1} \mathrm{R}^{n-1}, b_{1} \mathrm{R}^{n-1}, a_{2} \mathrm{R}^{n-2} \ldots$ all taken positively and a value of $R$ may be chosen so great that this sum does not exceed $k \mathrm{R}^{n}$ where $k$ is any finite quantity. Thus the sign of $u$ will be that of its first term if $\cos ^{2} n \theta>k^{2}$ and similarly for $v$ : we shall take $k=\frac{1}{2}$.

Divide $c$ into $4 n$ parts at the points where

$$
\theta=(2 r+1) \pi / 4 n \quad(r=0,1, \ldots 4 n-1)
$$

In the part of $c$ when $\theta$ rises from $(8 m-1) \pi / 4 n$ to $(8 m+1) \pi / 4 n \cos n \theta \$ 1 / \sqrt{ } 2$ and thus $u$ is positive while the sign of $v$ is at first - and at last + .

When $\theta$ rises from $(8 m+1) \pi / 4 n$ to $(8 m+3) \pi / 4 n, v$ is positive, but $u$ begins + and ends - .

When $\theta$ rises from $(8 m+3) \pi / 4 n$ to $(8 m+5) \pi / 4 n, u$ is always - .

When $\theta$ rises from $(8 m+5) \pi / 4 n$ to $(8 m+7) \pi / 4 n$, $v$ is always - .

Only in the second case is there any contribution to $(u, v ; c)$ and as this case occurs $n$ times contributing 1 each time we have

$$
(u, v ; c)=n \text {. }
$$

Hence $c$ must contain a point where $u, v$ vanish together, and the equation $f(z)=0$ must have a root.

The proof that there are exactly $n$ roots is now easy. 